\title{
Low-Dose Computed Tomography Filtering Using Geodesic Distances
}

\author{
Daniel A. Góes* \\ Centro Universitário Campo Limpo Paulista - UNIFACCAMP \\ Programa de Mestrado em Ciência da Computação
}

\author{
Nelson D. A. Mascarenhas \\ Universidade Federal de São Carlos - UFSCar \\ Departamento de Computação \\ Centro Universitário Campo Limpo Paulista - UNIFACCAMP \\ Programa de Mestrado em Ciência da Computação
}

In computed tomography, the noise can be described by a Compound Poisson distribution:

$$
Y=\sum_{n=1}^{N} \Phi_{n}
$$

where $N$ represents the number of detected photons, which obeys a Poisson distribution, and $\Phi_{n}$ are random variables, equally distributed, that model the energy of each photon, the probability distribution of $\Phi_{n}$ being derived from the incident spectrum.

Without spectral information of the incident beam, it is not possible to estimate the Compound Poisson distribution. In this work, we consider the model in which the detection process consists of counting the number of incident photons, thus obeying a typical Poisson distribution.

$$
\operatorname{Pr}\left(y_{i}=k \mid \lambda_{i}\right)=\frac{e^{-y_{i}} \lambda_{i}^{k}}{k !}
$$

Computed tomography is a technique that uses ionizing radiation, X-ray being the most common, to generate the scanned object image cuts. Due to the concern with the amount of radiation to which a patient is submitted, [1] and [2], lowdose computed tomography (LDCT) techniques have been developed to utilize the lowest radiation doses possible.

Tomographic projections are corrupted with signaldependent noise that can be modeled as Poisson noise, [2]. With the reduction in the number of photons, the degradation by noise is accentuated.

Buades et al. (2005) presented a new denoising method, Non-Local Means (NLM), for images corrupted by Gaussian noise using an auto similarity concept.

This work modifies the Non-Local Means using a Bayesian approach to filter Poisson noise. We evaluate our method by filtering sinograms obtained by LDCT, comparing the results with other methods.

\section{NOISE}

The X-ray emitter of a computed tomography scanner emits a poly-energetic beam, [3]. Materials have different attenuation coefficients for each energy band, [4], so the beam incident on a receiver can be described as polychromatic, [5].

\footnotetext{
* During the development of this work, D. A. Góes was with the Master's Program in Computer Science, UNIFACCAMP
}

where $\lambda_{i}$ is the average rate of photons arriving at the detector $i$ and $y_{i}$ is the observed photon quantity. $\operatorname{Pr}\left(y_{i}=k \mid \lambda_{i}\right)$ is the probability of $y_{i}$ to assume the value $k, k \in \mathbb{Z}^{+}$, with a photon rate of $\lambda_{i}$.

\section{NON-LOCAL MEANS}

Given an input noisy image $Y$, resulting from degradation of an original, noise-free image $X$, the Non-Local Means (NLM) method obtains an estimate $\hat{X}$, for the image $X$, where each pixel $\hat{x}_{i}$ is calculated by:

$$
\hat{x}_{i}=\frac{1}{w_{i}} \sum_{j \in B(i, r)} w_{i j} y_{j}
$$

where $0 \leq w_{i j} \leq 1, w_{i}=\sum_{j \in B(i, r)} w_{i j}, B(i, r)$ is the search square window of size $r$, centered on $i$, and the weights $w_{i j}$ are:

$$
w_{i j}=\exp \left(\frac{-1}{\sigma^{2}}\left\|y_{i}-y_{j}\right\|_{2, a}^{2}\right)
$$

$\sigma$ is a parameter that controls the intensity of the filter, $y_{i}$ and $y_{j}$ are vectors representing the similarity window centered on $i$ and $j$ respectively. 


\section{THEORETICAL FOUNDATION}

\section{A. Bayesian Approach}

Considering an ideal noise-free sinogram $X$ and a given sinogram $Y$ corrupted by Poisson noise, we can obtain an estimator of $X$ through a Bayesian approach.

$$
\operatorname{Pr}(X \mid Y)=\frac{\operatorname{Pr}(Y \mid X) \operatorname{Pr}(X)}{\operatorname{Pr}(Y)}
$$

In this model, the likelihood $\operatorname{Pr}(Y \mid X)$ is the noise distribution, and the posterior distribution $\operatorname{Pr}(X \mid Y)$ gives the estimator for the filtered sinogram. Computing this posterior distribution would require the usage of computationally expensive numerical techniques [6] [7]. Instead, we opted to use conjugate prior distributions, a property that if the prior distribution $\operatorname{Pr}(X)$ is conjugate to the likelihood distribution $\operatorname{Pr}(Y \mid X)$, then the posterior distribution is of the same type as the prior distribution.

In the case of Poisson likelihood, its conjugate is the Gamma distribution. Thus, if we assume that a neighborhood in the ideal sinogram obeys a Gamma distribution, then the neighborhood in the estimated sinogram will also obey a Gamma distribution. The Gamma distribution also has the very convenient property of being defined on the non-negative real line, assuring non-negative estimators for the rate of the Poisson distribution.

The geodesic distances used will be calculated between the a posteriori Gamma distributions, in the Bayesian model, using the statistical model of the tomographic reconstruction problem under Poisson noise.

1) Parameters Estimation: The posterior hyperparameters $\hat{\alpha}$ and $\hat{\beta}$ can be obtained from the corresponding hyperparameters of the a priori distribution $\alpha$ and $\beta$. These prior parameters are estimated from pre-filtering the sinogram with a $3 \times 3$ mean filter.

With the method of moments, the parameters are estimated from the mean $(\mu)$ and variance $\left(\sigma^{2}\right)$ calculated in a $3 \times 3$ window of the pre-filtered sinogram.

$$
\begin{aligned}
\alpha & =\frac{\mu^{2}}{\sigma^{2}} \\
\beta & =\frac{\mu}{\sigma^{2}}
\end{aligned}
$$

The posterior parameters are also estimated through the method of moments:

$$
\begin{aligned}
& \hat{\alpha}=\alpha+\sum_{i=1}^{n} y_{i} \\
& \hat{\beta}=\beta+n
\end{aligned}
$$

where $\sum_{i=1}^{n} y_{i}$ is the sum of the projection values in the noisy sinogram window.

\section{B. Geodesic distances}

Geodesic distance is the minimum distance between two points passing through a surface. A Riemannian manifold is a generalization of a surface used for calculating these types of distances.
Rao [8] described the parametric space of a probability distribution family as a Riemannian manifold and derived geodesic distances using the Kullback Leiber divergence. These distances could then be used as similarity metrics between two different distributions belonging to the same probability family.

Menéndez et al. [9] proposed a general method for generating geodesic distances for $(h, \phi)$-entropies, introduced by Salicru et al. [10]. The parametric space manifold is given by:

$$
H_{\phi}^{h}=h\left[\int_{I} \phi\left(f_{P}(x ; \theta)\right) d x\right]
$$

where $\theta$ is the hyperparameter vector, $f_{P}(x ; \theta)$ the probability density function and $I$ its support range, $(0, \infty)$ for the Gamma distributions.

The geodesic distance is then defined by:

$$
d\left(\theta_{a}, \theta_{b}\right)=\left|\int_{\theta_{a}}^{\theta_{b}}\left[\sum_{i=1}^{M} \sum_{j=1}^{M} g_{i j}(\theta) d \theta_{i} d \theta_{j}\right]^{\frac{1}{2}}\right|
$$

where $\theta_{a}$ and $\theta_{b}$ are the hyperparameter vectors and $g_{i j}$ is the Hessian of $H_{\phi}^{h}$.

$$
\begin{aligned}
& g_{i j}(\theta)= \\
& \quad h^{\prime \prime}\left[\int_{I} \phi\left(f_{P}(x ; \theta)\right) d x\right] \int_{I} \phi^{\prime}\left(f_{P}(x ; \theta)\right) \frac{\partial f_{P}(x ; \theta)}{\partial \theta_{i}} d x \\
& \times \int_{I} \phi^{\prime}\left(f_{P}(x ; \theta)\right) \frac{\partial f_{P}(x ; \theta)}{\partial \theta_{j}} d x \\
& \quad+h^{\prime}\left[\int_{I} \phi\left(f_{P}(x ; \theta)\right) d x\right] \\
& \times \int_{I} \phi^{\prime \prime}\left(f_{P}(x ; \theta)\right) \frac{\partial f_{P}(x ; \theta)}{\partial \theta_{i}} \frac{\partial f_{P}(x ; \theta)}{\partial \theta_{j}} d x
\end{aligned}
$$

TABLE I

$(h, \phi)$-ENTROPIES AND THEIR RESPECTIVE $h(x), \phi(x)$ FUNCTIONS

\begin{tabular}{|c||c|c|}
\hline$(h, \phi)$-entropy & $h(x)$ & $\phi(x)$ \\
\hline \hline Arimoto & $\left(x^{s}-1\right) /(s-1)$ & $x^{\frac{1}{s}}, s>0, s \neq 1$ \\
\hline Havrda-Charvát & $x$ & $\left(x^{s}-x\right) /(s-1), s>0, s \neq 1$ \\
\hline Rényi & $\log (x) /(s-1)$ & $x^{s}, s>0, s \neq 1$ \\
\hline Shannon & $x$ & $-x \log (x)$ \\
\hline Tsallis & $(x-1) /(s-1)$ & $x^{s}, s>0, s \neq 1$ \\
\hline
\end{tabular}

This work uses the $(h, \phi)$-entropies described in the table I. For the Tsallis entropy, the distances calculated were equivalent to those obtained by the Havrda-Charvát entropy, despite the different values of $g_{i j}$. Thus, the results are only presented for the Havrda-Charvát entropy.

For the Gamma distribution, it was only possible to find a closed-form solution for the geodesic distance using Shannon's entropy. For the other entropies, it was possible to calculate the terms $g_{i j}$, so numerical integration was used to calculate the final distance $d\left(\theta_{a}, \theta_{b}\right)$.

The Shannon geodesic distance for the Gamma distribution is given by: 


$$
\begin{aligned}
& d\left(\theta_{1}, \theta_{2}\right)= \\
& \quad \mid\left(\beta_{1}-2 \alpha_{1} \log \left(\beta_{1}\right)-\beta_{1} \log \left(\beta_{1}\right)+\log \Gamma\left(\alpha_{1}\right)\right)^{\frac{1}{2}} \\
& -\left(\beta_{2}-2 \alpha_{2} \log \left(\beta_{2}\right)-\beta_{2} \log \left(\beta_{2}\right)+\log \Gamma\left(\alpha_{2}\right)\right)^{\frac{1}{2}} \mid
\end{aligned}
$$

\section{Proposed Geodesic Filtering Method}

The proposed Poisson noise reduction filter is based on Evangelista's [11] approach. In our method, the NLM is modified to use geodesic distances between Gamma distributions, where the parameters are estimated for the posterior distribution using the Bayesian approach.

The comparison between similarity windows is made by calculating the geodesic distance between each element, with the final distance being the sum of these distances. The weight $w_{i j}$ from equation 4 is replaced by:

$$
w_{i j}=\exp \left(\frac{-\sum_{P} d\left(\theta_{i-p}, \theta_{j-p}\right)}{\sigma^{2}}\right)
$$

where $P$ indexes all elements of the similarity window.

\section{Evaluation}

\section{A. Data Set}

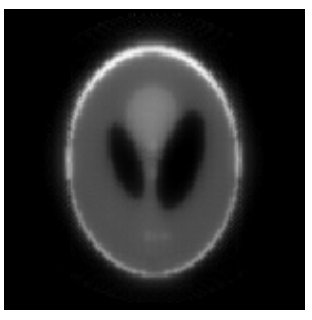

(a) Shepp-Logan

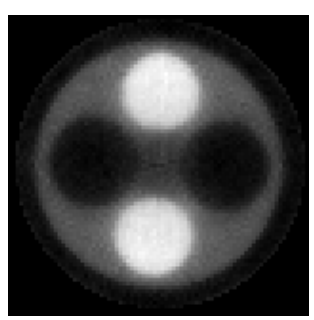

(c) Symmetric

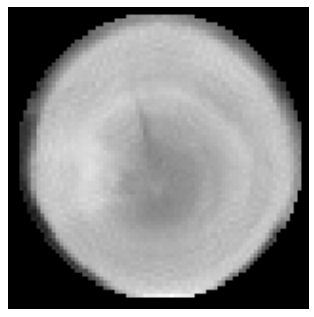

(e) Wood 1

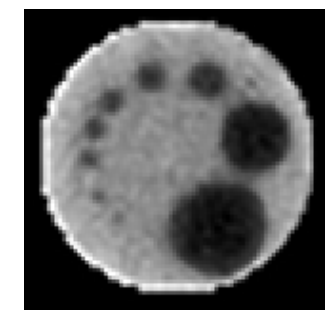

(b) Asymmetric

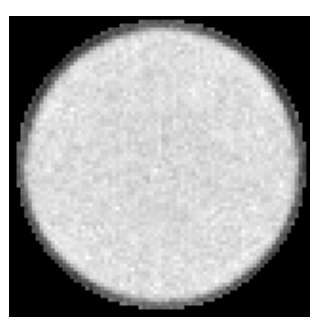

(d) Homogeneous

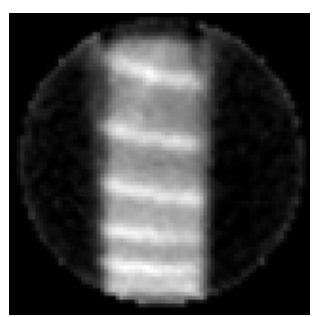

(f) Wood 2
Fig. 1. Noise-Free Reference Images Set Reconstructed by Projection onto Convex Sets (POCS)
The data set used in this work is composed of the SheppLogan phantom (simulating a cut of the human brain) as well as five real sinograms phantoms kindly provided by Prof. Dr. Paulo Estevão Cruvinel from EMBRAPA-CNPDIA [12].

The noisy sinograms were acquired from 3 seconds exposures per projection point, while phantoms considered noisefree were acquired from 20 seconds exposures per projection point.

The X-ray energy used was $59.5 \mathrm{keV}$, the tomographic scanning was performed in an angular rotation of $180^{\circ}$ with a translation of $1 \mathrm{~mm}$.

The noisy Shepp-Logan sinogram was simulated by calculating the Radon transform of the image, and Poisson noise was introduced in the projections.

The Asymmetric phantom was acquired from a $96 \mathrm{~mm}$ diameter PEXIGLASS $\cap$ cylinder with ten holes filled with air, with sizes ranging from $0.5 \mathrm{~mm}$ to $5 \mathrm{~mm}$. The projection matrix is $100 \times 100$.

The Symmetrical phantom was acquired from a $76 \mathrm{~mm}$ diameter PEXIGLASS $\AA$ cylinder with four holes, two filled with aluminum and 2 with air. The projection matrix is $80 \times 80$.

The Homogeneous phantom was acquired from a $2 \mathrm{~mm}$ thick and $76 \mathrm{~mm}$ of diameter PEXIGLASS $\AA$ cylinder filled with water. The projection matrix is $80 \times 80$.

The Wood 1 phantom was acquired from scanning a $70 \mathrm{~mm}$ diameter Eucalyptus Saligna trunk. The projection matrix is $76 \times 76$.

The Wood 2 phantom was acquired from a $50 \mathrm{~mm} \times$ $50 \mathrm{~mm} \times 50 \mathrm{~mm}$ Pinus Elliottii trunk. The projection matrix is $62 \times 62$.

\section{B. Evaluated Methods}

In this work, we evaluate the proposed method using the geodesic distances for the following entropies:

1) Shannon, the comparison is made with the closed-form solution for the distance.

2) Arimoto, the comparison is numeric and with the entropy parameter $s=1.1$.

3) Havrda-Charvát, the comparison is numeric with the parameter $s=0.8$.

4) Rényi, the comparison is numeric with the parameter $s=0.1$.

We compared the proposed method with the original NLM applied in the Anscombe domain (NLM), the Poisson-NLM method [13] (P-NLM), the SP-NLM method [14] (SP-NLM), the stochastic Gamma NLM method [11] (G-NLM), and the BM3D [15] method applied to the Anscombe domain (BM3D).

The Stochastic Poisson NLM (SP-NLM) is an algorithm developed by Bindilatti and Mascarenhas [14] that modifies the NLM by replacing the Euclidean distance with stochastic distances between Poisson distributions. The distances used are Bhattacharyya, Hellinger, Kullback-Leibler, and Rényi. Evangelista [11] proposed an alteration to the SP-NLM, using a Bayesian approach by replacing stochastic distances between Poisson distributions with stochastic distances between Gamma distributions, conjugate of the Poisson. The 
distances used are Bhattacharyya, Hellinger, Kullback-Leibler, and Rényi.

\section{Filtering Parameters}

The NLM based methods were evaluated with search windows varying from $5 \times 5$ to $11 \times 11$. The similarity windows vary from $5 \times 5$ up to the size of the search window being used.

Except for Stochastic Poisson NLM, that calculates the $\sigma$ parameter automatically, in the other methods, the $\sigma$ parameter varied between 0.1 and 0.5. Except for the homogeneous and Shepp-Logan phantoms that ranged between 0.2 and 0.6 .

\section{Reconstruction and Evaluation}

The sinograms are reconstructed with both FBP and POCS [16] methods. The resulting filtered images are compared with the image obtained by the noise-free sinograms using two similarity metrics, PSN and SSIM [17].

\section{RESULTS}

The results presented in tables II through VII are the best ones obtained by each method in each different metric, with the best overall value presented in bold and the second-best in italic. For SP-NLM and G-NLM, only the best performing stochastic distance for each metric is considered. Figure 2 shows some of the filtered Shepp-Logan images.

The standard NLM in the Anscombe domain was not able to achieve comparable results to the other evaluated methods.

The P-NLM method only outperformed the proposed method, in all metrics, in the Symmetric phantom. While in Shepp-Logan and Wood 2, it achieved best results only in one metric each, PSNR in FBP and PSNR in POCS, respectively.

Except for the Homogeneous phantom, the proposed method achieved best results than SP-NLM in virtually all metrics (there was only one with the same result in the Asymmetric phantom).

When comparing our method to the G-NLM, in the Asymmetric phantom, both achieved similar results.

In the Shepp-Logan, our method had best results in the two metrics under FBP, the G-NLM had a best PSNR in POCS, and they had the same result in the last metric. While in the Homogeneous phantom, the G-NLM achieved a superior result in only one metric, PSNR in FBP, with the proposed method having best results in the other three.

For the other three phantoms, Symmetric, Wood 1 and Wood 2, our method outperformed G-NLM in all four metrics.

In comparison to the $\mathrm{BM} 3 \mathrm{D}$, only in the Asymmetric phantom, the BM3D outperformed the proposed method in all metrics. While in the Homogeneous Phantom, our method outperformed BM3D, also in all metrics.

When comparing the PSNR metric with FBP reconstruction, our method achieved best results in five of the tests, having an inferior result only in the Asymmetric phantom.

In the SSIM metric with FBP reconstruction, the BM3D had best results in three phantoms, while our method had a superior result in one phantom, the other two both had the same results.
In POCS reconstruction, both had similar results, with each surpassing the other in half the data set for the PSNR metric, and in SSIM, each method achieved a best result twice and having the same result in two phantoms.

TABLE II

SHEPP-LOGAN RESULTS

\begin{tabular}{|c||c|c|c|c|}
\hline \multirow{2}{*}{ Methods } & \multicolumn{2}{c|}{ FBP } & \multicolumn{2}{c|}{ POCS } \\
\cline { 2 - 5 } & $\begin{array}{c}\text { PSNR } \\
(\mathrm{dB})\end{array}$ & SSIM & $\begin{array}{c}\text { PSNR } \\
(\mathrm{dB})\end{array}$ & SSIM \\
\hline \hline Noisy & 14.51 & 0.25 & 20.22 & 0.62 \\
\hline Shannon & 20.98 & 0.76 & 22.11 & $\underline{0.89}$ \\
\hline Arimoto & 21.14 & $\mathbf{0 . 8 1}$ & $\underline{22.14}$ & $\mathbf{0 . 9 0}$ \\
\hline Havrda Charvát & 21.09 & $\underline{0.80}$ & 21.68 & $\mathbf{0 . 9 0}$ \\
\hline Rényi & 21.07 & $\underline{0.80}$ & 21.67 & $\mathbf{0 . 9 0}$ \\
\hline NLM & 20.36 & 0.61 & 21.86 & 0.86 \\
\hline P-NLM & $\mathbf{2 1 . 2 0}$ & 0.72 & 21.77 & 0.88 \\
\hline SP-NLM & 18.84 & 0.73 & 18.33 & 0.86 \\
\hline G-NLM & 20.97 & $\underline{0.80}$ & $\mathbf{2 2 . 2 0}$ & $\mathbf{0 . 9 0}$ \\
\hline BM3D & 21.00 & $\mathbf{0 . 8 1}$ & 21.30 & $\underline{0.89}$ \\
\hline
\end{tabular}

TABLE III

ASYMMETRIC RESULTS

\begin{tabular}{|c||c|c|c|c|}
\hline \multirow{2}{*}{ Methods } & \multicolumn{2}{c|}{ FBP } & \multicolumn{2}{c|}{ POCS } \\
\cline { 2 - 5 } & $\begin{array}{c}\text { PSNR } \\
(\mathrm{dB})\end{array}$ & SSIM & $\begin{array}{c}\text { PSNR } \\
(\mathrm{dB})\end{array}$ & SSIM \\
\hline \hline Noisy & 16.52 & 0.52 & 24.27 & 0.85 \\
\hline Shannon & 20.89 & 0.69 & 28.85 & $\underline{0.91}$ \\
\hline Arimoto & 21.18 & $\underline{0.70}$ & 29.11 & $\underline{0.91}$ \\
\hline Havrda Charvát & 21.24 & $\underline{0.70}$ & $\underline{29.15}$ & $\underline{0.91}$ \\
\hline Rényi & 21.16 & $\underline{0.70}$ & $\underline{29.15}$ & $\underline{0.91}$ \\
\hline NLM & 20.10 & 0.67 & 26.91 & 0.90 \\
\hline P-NLM & 20.81 & 0.68 & 28.85 & 0.90 \\
\hline SP-NLM & 20.96 & 0.69 & 28.72 & $\underline{0.91}$ \\
\hline G-NLM & $\underline{21.26}$ & $\underline{0.70}$ & 29.14 & $\underline{0.91}$ \\
\hline BM3D & $\mathbf{2 1 . 4 6}$ & $\mathbf{0 . 7 2}$ & $\mathbf{2 9 . 6 7}$ & $\mathbf{0 . 9 2}$ \\
\hline
\end{tabular}

\section{CONCLUSION}

We developed a method to denoise data corrupted by Poisson noise. We used a Bayesian strategy to alter the NonLocal Means replacing the Euclidean distance by a geodesic statistical distances between a posteriori Gamma distributions. Low-dose computed tomography data were used to evaluate our method.

Due to mathematical simplicity, the proposed method assumes that the original noise-free sinogram obeys a Gamma distribution, since it is the conjugate to the Poisson distribution (used to model the noise). The results obtained show that the approach of using the combined Gamma and Poisson distributions is feasible.

The usage of geodesic distances instead of stochastic ones improved the overall results, obtaining, in general, the best 
TABLE IV

SYMMETRIC RESULTS

\begin{tabular}{|c||c|c|c|c|}
\hline \multirow{2}{*}{ Methods } & \multicolumn{2}{c|}{ FBP } & \multicolumn{2}{c|}{ POCS } \\
\cline { 2 - 5 } & $\begin{array}{c}\text { PSNR } \\
(\mathrm{dB})\end{array}$ & SSIM & $\begin{array}{c}\text { PSNR } \\
(\mathrm{dB})\end{array}$ & SSIM \\
\hline \hline Noisy & 20.54 & 0.45 & 27.51 & 0.80 \\
\hline Shannon & $\underline{27.88}$ & $\mathbf{0 . 7 2}$ & 34.42 & $\mathbf{0 . 9 5}$ \\
\hline Arimoto & 27.81 & $\mathbf{0 . 7 2}$ & 33.91 & $\mathbf{0 . 9 5}$ \\
\hline Havrda Charvát & 27.70 & 0.71 & 34.42 & $\underline{0.94}$ \\
\hline Rényi & 27.77 & $\mathbf{0 . 7 2}$ & 34.50 & $\mathbf{0 . 9 5}$ \\
\hline NLM & 26.27 & 0.63 & 33.35 & 0.93 \\
\hline P-NLM & $\mathbf{2 8 . 0 0}$ & $\mathbf{0 . 7 2}$ & $\mathbf{3 4 . 5 7}$ & $\mathbf{0 . 9 5}$ \\
\hline SP-NLM & 25.27 & 0.67 & 32.37 & 0.92 \\
\hline G-NLM & 27.81 & $\mathbf{0 . 7 2}$ & 34.43 & $\mathbf{0 . 9 5}$ \\
\hline BM3D & 27.34 & $\mathbf{0 . 7 2}$ & 33.27 & $\mathbf{0 . 9 5}$ \\
\hline
\end{tabular}

TABLE V

HomogeneOUS RESULTS

\begin{tabular}{|c||c|c|c|c|}
\hline \multirow{2}{*}{ Methods } & \multicolumn{2}{c|}{ FBP } & \multicolumn{2}{c|}{ POCS } \\
\cline { 2 - 5 } & $\begin{array}{c}\text { PSNR } \\
(\mathrm{dB})\end{array}$ & SSIM & $\begin{array}{c}\text { PSNR } \\
(\mathrm{dB})\end{array}$ & SSIM \\
\hline Noisy & 13.35 & 0.12 & 15.47 & 0.42 \\
\hline Shannon & 18.62 & 0.32 & 19.98 & 0.66 \\
\hline Arimoto & 18.92 & 0.33 & 20.00 & 0.66 \\
\hline Havrda Charvát & 18.93 & $\underline{0.34}$ & $\underline{20.82}$ & $\underline{0.70}$ \\
\hline Rényi & 18.84 & 0.32 & 20.69 & 0.68 \\
\hline NLM & 17.35 & 0.21 & 18.54 & 0.57 \\
\hline P-NLM & 18.35 & 0.29 & 19.56 & 0.64 \\
\hline SP-NLM & $\underline{19.26}$ & $\mathbf{0 . 3 9}$ & $\mathbf{2 3 . 6 9}$ & $\mathbf{0 . 7 4}$ \\
\hline G-NLM & $\mathbf{1 9 . 3 6}$ & 0.33 & 20.53 & 0.69 \\
\hline BM3D & 16.53 & 0.29 & 19.12 & 0.66 \\
\hline
\end{tabular}

TABLE VI

WOOD 1 RESULTS

\begin{tabular}{|c||c|c|c|c|}
\hline \multicolumn{1}{|c|}{ Methods } & \multicolumn{2}{c|}{ FBP } & \multicolumn{2}{c|}{ POCS } \\
\cline { 2 - 5 }$(\mathrm{dB})$ & SSIM & $\begin{array}{c}\text { PSNR } \\
(\mathrm{dB})\end{array}$ & SSIM \\
\hline Noisy & 21.91 & 0.68 & 34.08 & 0.91 \\
\hline Shannon & $\underline{30.66}$ & 0.83 & $\underline{38.48}$ & $\underline{0.96}$ \\
\hline Arimoto & 29.11 & $\underline{0.84}$ & 38.46 & $\mathbf{0 . 9 7}$ \\
\hline Havrda Charvát & $\mathbf{3 0 . 6 9}$ & $\underline{0.84}$ & 38.12 & $\mathbf{0 . 9 7}$ \\
\hline Rényi & 30.64 & $\underline{0.84}$ & $\underline{38.48}$ & $\mathbf{0 . 9 7}$ \\
\hline NLM & 28.44 & 0.82 & 37.75 & $\underline{0.96}$ \\
\hline P-NLM & 28.38 & 0.81 & 37.46 & $\underline{0.96}$ \\
\hline SP-NLM & 23.32 & 0.70 & 21.38 & 0.92 \\
\hline G-NLM & 30.58 & 0.83 & 38.05 & $\underline{0.96}$ \\
\hline BM3D & 29.25 & $\mathbf{0 . 8 5}$ & $\mathbf{3 8 . 7 5}$ & $\mathbf{0 . 9 7}$ \\
\hline
\end{tabular}

results among the methods based on NLM and close results to BM3D.

Using Shannon's entropy, we were able to find a closedform geodesic distance for the Gamma distribution. To our knowledge, no previous work has published a similar solution
TABLE VII

WOOD 2 RESULTS

\begin{tabular}{|c||c|c|c|c|}
\hline \multirow{2}{*}{ Methods } & \multicolumn{2}{c|}{ FBP } & \multicolumn{2}{c|}{ POCS } \\
\cline { 2 - 5 } & $\begin{array}{c}\text { PSNR } \\
(\mathrm{dB})\end{array}$ & SSIM & $\begin{array}{c}\text { PSNR } \\
(\mathrm{dB})\end{array}$ & SSIM \\
\hline \hline Noisy & 19.88 & 0.56 & 21.66 & 0.69 \\
\hline Shannon & $\underline{26.33}$ & $\underline{0.68}$ & 30.05 & $\underline{0.91}$ \\
\hline Arimoto & 26.21 & 0.67 & 30.00 & $\underline{0.91}$ \\
\hline Havrda Charvát & $\mathbf{2 6 . 3 9}$ & $\underline{0.68}$ & 30.62 & $\underline{0.91}$ \\
\hline Rényi & 26.23 & 0.67 & 30.45 & 0.90 \\
\hline NLM & 24.64 & 0.68 & 28.55 & 0.88 \\
\hline P-NLM & 25.76 & 0.67 & $\underline{30.79}$ & $\underline{0.91}$ \\
\hline SP-NLM & 18.02 & 0.47 & 22.41 & 0.74 \\
\hline G-NLM & 25.35 & 0.65 & 29.28 & 0.89 \\
\hline BM3D & 24.80 & $\mathbf{0 . 7 2}$ & $\mathbf{3 1 . 4 3}$ & $\mathbf{0 . 9 2}$ \\
\hline
\end{tabular}

for this model.

For the other entropies, in which it was not possible to obtain a closed-form solution for the geodesic distance, numerical integration was used to calculate the final distance, with no perceived loss in the quality of the results when compared to the distance using Shannon's entropy.

It was observed that the quality of the results varies considerably, depending on the value chosen for the $\sigma$ parameter (equation 12). As future work, there is the possibility of automatically configuring this parameter. Another option is to configure this parameter in an adaptive way, where $\sigma$ assumes different values for each pixel being filtered.

Using geodesic distances between conjugated distributions to the noise model has the potential to be adapted for other data domains, as synthetic aperture radar images and other forms of medical images like magnetic resonance imaging.

The geodesic distance model proved to be effective when comparing computed tomography sinogram patches. Thus, these distances could be used in the design of descriptors for pattern recognition applications in CT.

This work was published in Digital Signal Processing [18].

The proposed method code was published on the CodeOcean platform at the link:

https://doi.org/10.24433/CO.baa5e6c4-d046-4e20-8eb29077c1ce0dee

\section{ACKNOWLEDGMENT}

This work was financed in part by the Coordenação de Aperfeiçoamento de Pessoal de Nível Superior - Brasil (CAPES) - Finance Code 001.

We thank Dr. Paulo Estevão Cruvinel of EMBRAPACNPDIA who provided the real phantoms data used in this work.

The work of N. D. A. Mascarenhas was supported by $\mathrm{CNPq}$, Brazil, through a scholarship under Grant Process 306742/2017-9. 


\section{REFERENCES}

[1] N. Savage, "Medical imagers lower the dose," IEEE Spectrum, vol. 47, no. 3, pp. 14-16, 2010.

[2] J. Z. Liang et al., "Guest editorial low-dose ct: What has been done, and what challenges remain?" IEEE Transactions on Medical Imaging, vol. 36, no. 12, pp. 2409-2416, Dec 2017

[3] I. A. Elbakri and J. A. Fessler, "Efficient and accurate likelihood for iterative image reconstruction in x-ray computed tomography," in Medical Imaging 2003: Image Processing, M. Sonka and J. M. Fitzpatrick, Eds., vol. 5032, May 2003, pp. 1839-1850.

[4] L. F. Granato, P. E. Cruvinel, F. Cassaro, and S. Crestana, "A technique for improvement of linear attenuation coefficient maps obtained by means of $\mathrm{x}$-ray tomography in multiple energies," in Proceedings SIBGRAPI'98. International Symposium on Computer Graphics, Image Processing, and Vision (Cat. No.98EX237), Oct 1998, pp. 62-68.

[5] R. Gu and A. Dogandžić, "Blind X-ray ct image reconstruction from polychromatic poisson measurements," IEEE Transactions on Computational Imaging, vol. 2, no. 2, pp. 150-165, June 2016.

[6] A. Webb and K. Copsey, Statistical Pattern Recognition. Wiley, 2011.

[7] V. Šmídl, V. Smidl, and A. Quinn, The Variational Bayes Method in Signal Processing, ser. Signals and Communication Technology. Springer, 2006.

[8] C. R. Rao, "Information and accuracy attainable in the estimation of statistical parameters," Bulletin of Calcutta Mathematical Society, vol. 37, no. 3, pp. 81-91, 1945.

[9] M. L. Menéndez, D. Morales, L. Pardo, and M. Salicrú, “( $h, \Phi)$-entropy differential metric," Applications of Mathematics, vol. 42, no. 2, pp. 8198, 1997.

[10] M. Salicrú, M. Menéndez, D. Morales, and L. Pardo, "Asymptotic distribution of $(h, \phi)$-entropies," Communications in Statistics - Theory and Methods, vol. 22, no. 7, pp. 2015-2031, 1993.

[11] R. C. Evangelista, "Abordagens bayesianas não-locais para filtragem de ruído poisson em imagens tomograficas com baixas taxas de contagem utilizando distâncias estocásticas," Master's thesis, Centro Universitário de Campo Limpo Paulista - UNIFACCAMP, 2017.

[12] R. J. Geraldo, L. M. V. Cura, P. E. Cruvinel, and N. D. A. Mascarenhas, "Low dose CT filtering in the image domain using MAP algorithms," IEEE Transactions on Radiation and Plasma Medical Sciences, vol. 1, no. 1, pp. 56-67, 2017.

[13] C. Deledalle, F. Tupin, and L. Denis, "Poisson NL means: Unsupervised non local means for Poisson noise," Proceeding of the International Conference on Image Processing, pp. 801 - 804, 2010.

[14] A. A. Bindilatti and N. D. A. Mascarenhas, "A nonlocal Poisson denoising algorithm based on stochastic distances," IEEE Signal Processing Letters, vol. 20, no. 11, pp. 1010-1013, Nov 2013.

[15] K. Dabov, A. Foi, V. Katkovnik, and K. Egiazarian, "Image denoising by sparse 3-d transform-domain collaborative filtering," IEEE Transactions on Image Processing, vol. 16, no. 8, pp. 2080-2095, 2007.

[16] F. V. Salina and N. D. A. Mascarenhas, "A hybrid estimation theoreticpocs method for tomographic image reconstruction," in XVIII Brazilian Symposium on Computer Graphics and Image Processing (SIBGRAPI'05), Oct 2005, pp. 220-224.

[17] Z. Wang, A. C. Bovik, H. R. Sheikh, and E. P. Simoncelli, "Image quality assessment: from error visibility to structural similarity," IEEE Transactions on Image Processing, vol. 13, no. 4, pp. 600-612, April 2004.

[18] D. A. Góes and N. D. Mascarenhas, "Poisson denoising under a Bayesian nonlocal approach using geodesic distances with low-dose CT applications," Digital Signal Processing, vol. 106, p. 102835, Nov 2020.

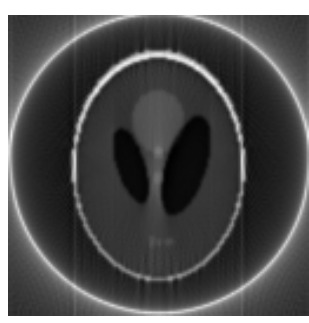

Noise-Free

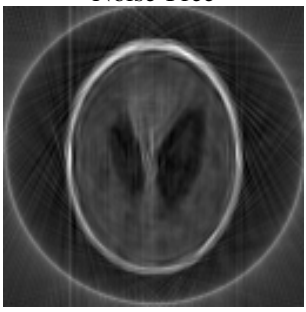

Shannon

$11 \times 11 ; 11 \times 11 ; 0.60$

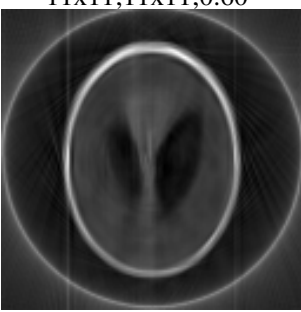

Havrda Charvát $15 \times 15 ; 9 \times 9 ; 0.60$

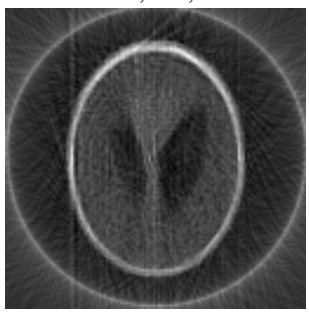

NLM

$15 \times 15 ; 11 \times 11 ; 0.60$

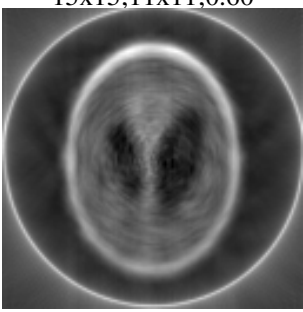

SP-NLM $7 \times 7 ; 7 \times 7$

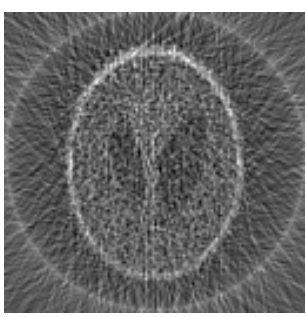

Noisy

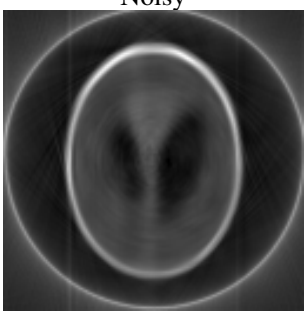

Arimoto

$11 \times 11 ; 9 \times 9 ; 0.65$

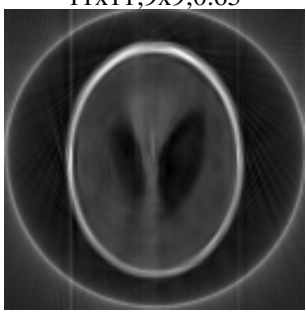

Rényi

15x15;9x9;0.60

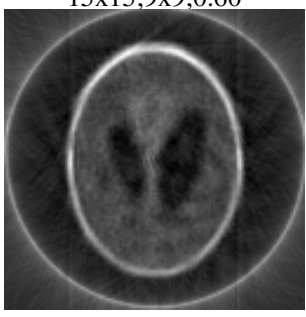

P-NLM

$13 \times 13 ; 11 \times 11: 0.4$

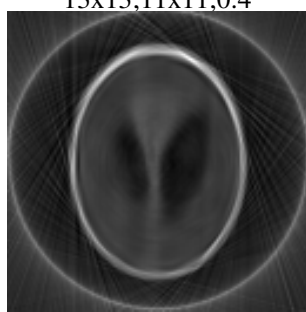

G-NLM

$11 \times 11 ; 11 \times 11 ; 0.50$

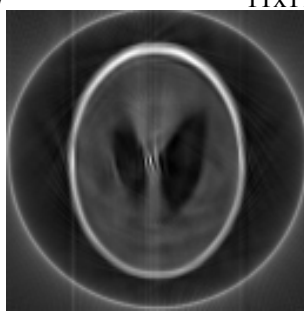

BM3D

Fig. 2. Images from Shepp-Logan Results for FBP Reconstruction 